\title{
Minireview
}

\section{AN ANALYTICAL APPROACH TO THE THIRD BODY MODELLING IN FRETTING WEAR CONTACT: A MINIREVIEW}

\author{
Ivan I. Argatov ${ }^{1}$, Young Suck Chai ${ }^{2}$ \\ ${ }^{1}$ Institut für Mechanik, Technische Universität Berlin, Berlin, Germany \\ ${ }^{2}$ School of Mechanical Engineering, Yeungnam University, Gyeongsan, Korea

\begin{abstract}
In fretting wear contact, the third body is defined as the wear debris bed between two contacting bodies. The problem of third-body modelling is considered from a point of view of contact mechanics. This paper is restricted to a discussion of recent developments in analytical modelling of fretting wear contact.
\end{abstract}

Key words: Fretting wear, Gross slip regime, Partial slip regime, Third-body layer, Asymptotic modeling, Self-similarity

\section{INTRODUCTION}

Fretting wear is a complex mechanical process of surface damage accumulation in loaded contacts of tribosystems, which are subject to oscillatory tangential displacements at low amplitude [1]. Fretting fatigue and wear are frequently encountered in critical engineering sectors, such as nuclear [2,3] and aerospace [4].

In contrast to sliding wear, which is realized, e.g., in pin-on-disc experiments, in fretting wear, which takes place, for instance, in ball-on-disc fretting tests, the wear debris experiences difficulties in escaping from the contact interface established between two contacting bodies (usually called first bodies), thereby forming a debris bed or a third-body layer [5]. Taking for granted that the third body is formed from first-body particles, its formation, flow, and elimination, in turn, influence the contact conditions between the first bodies as well as the surface degradation process.

In recent years, a number of numerical studies have been carried out to simulate the evolution of third body under different testing scenarios [6-8]. On the other hand, much attention has been also paid to constitutive modelling of third body $[9,10]$. Nevertheless, there is a lack of simple analytical models of contact interaction via a third body layer that can describe at least approximately all main aspects of the fretting wear contact, including

Received January 03, 2020 / Accepted February 08, 2021

Corresponding author: Young Suck Chai

Yeungnam University, School of Mechanical Engineering, Gyeongsan 712-749, South Korea

E-mail: yschai@yu.ac.kr 
the modification of the contact pressures, the expansion of the contact area, the growth of the third-body layer, and the accumulation of the worn material.

In the present minireview, we consider the problem of third-body modelling from a viewpoint of contact mechanics and adopting a continuum mechanics approach.

\section{MAIN CONTACT PARAMETERS}

External loading is apparently one of the major factors that govern the process of wear damage. When two bodies are brought into contact under a normal load, $P$, a contact interface is established, which redistributes the load over a contact region, $\omega$, with some contact pressure density, $p(x)$. The equation of equilibrium in the normal direction implies that

$$
P=\int_{\omega} p(x) d x
$$

whereas the distribution of contact pressures $p(x)$ strongly depends on the contact geometry and the mechanical properties of the contact bodies [11].

In fretting contact, the contact interface is subjected to the additional tangential loading, which often can be regarded as oscillating of a relatively low amplitude, $F_{T}$. This means that, strictly speaking, the fretting contact problem cannot be longer considered as monotonicallyevolving, though the oscillatory nature of tangential loading allows to introduce average characteristics for the evolution of tangential stresses per one-cycle loading, such as the dissipated friction energy, $\Delta E_{\mathrm{d}}$. In the simplest case, when the coefficient of friction, $\mu$, is assumed to be constant, the quantity $\Delta E_{\mathrm{d}}$ will be proportional to $\mu$, which is a consequence of the Coulomb law of friction.

The dependence of $\Delta E_{\mathrm{d}}$ on the tangential force amplitude $F_{T}$ is strongly influenced by the fretting regime [12], which, to simplify the consideration, can be classified into two primary categories: $(i)$ gross-slip and $(i i)$ partial-slip. While under the gross-slip conditions, the linear proportionality between $\Delta E_{\mathrm{d}}$ and $F_{T}$ can be adopted without sacrificing accuracy, the case of partial contact, when the contact zone $\omega$ is subdivided into a stick zone and a slip zone, requires a special consideration [13-15].

In Hertzian contact mechanics, the contact configuration is usually described in terms of the gap function, $\varphi(x)$, which is defined as the variable distance between the contacting surfaces measured along the normal to the joint contact plane in the undeformed configuration. (We note that, specifically, this definition applies to non-conformal contacts [16], when the normalization condition $\varphi(0)=0$ is usually applied.) The effect of wear manifests itself in the variation of the contact geometry [17], which under fretting contact conditions can be conveniently described by the variable gap function $\varphi_{N}(x)$, where $N$ is the number of fretting cycles (for details see, e.g., $[18,19]$ ). As such, the change between $\varphi_{N}(x)$ and $\varphi_{N+1}(x)$ will be attributed to the effect of wear, and the total wear volume increment in the $N$-th cycle can be evaluated as

$$
\Delta V_{N}=\int_{\omega_{N}} \Delta \varphi_{N}(x) d x
$$

where $\omega_{N}$ is the current contact region. We underline that the quantity defined by Eq. (2) accounts for contributions from both first bodies.

In sliding wear conditions, it is customary to assume that the worn material escapes from the contact interface and, thus, the wear debris does not interfere with the contact 
conditions existing at the contact interface after the moment of wear damage production. Generally speaking, the material removed from one contacting body can be added on the other one, and this picture is natural at the micro- and nanoscales. In what follows, we consider the fretting wear contact from a macroscale point of view, and therefore, the wear process for first bodies will be regarded as an irreversible operation.

\section{THIRD BODY MODELLING}

When the wear debris is retained within the contact region, the contact modelling should take into account the fact that the direct contact between the surfaces of the first bodies is lost partially or completely. In principle, the effect of relatively large wear particles trapped between the contacting surfaces can be modelled using a "discrete" approach (see, e.g., [2022]). The term "third body", as it is used hereafter, refers to circumstances, where the wear debris forms a third-body layer, which can be looked at from a continuum mechanics point of view.

A general method for modelling friction, wear, frictional heat, and mass efflux from a contact interface was developed by Zmitrowicz [23] (see also [24]) in the broad framework of the thermomechanical theory. The third-body layer effect was suggested to be modeled as an interfacial layer of wear products, assuming that its thickness is negligibly small, and the deformation of the interfacial layer is described as that of a two-dimensional micropolar material medium. A detailed review of continuum constitutive models of wear debris was given by Zmitrowicz [10] with a focus on micropolar thermoelastic layers. However, while the micropolar layer model suits well for modelling thin lubricating films containing wear debris in sliding conditions, there is an experimental evidence [3,25] that the third-body layer thickness varies significantly in fretting wear.

The third-body layer can be properly characterized by its variable thickness, $h(x, N)$, defined inside the contact region $\omega_{N}$ and subject to the initial condition $h(x, 0) \equiv 0$. Thus, assuming that the contact interaction between the two first bodies occurs through the thirdbody layer, we come to the question of modelling the deformation response of third body. As a first approximation, a Winkler-type model can be adopted to describe the normal deformation, which (in the asymptotics of a thin elastic layer [26,27]) besides the layer thickness also requires the aggregate elastic modulus of third body, $E_{A}^{\mathrm{tb}}$. The latter quantity determines the third-body layer deformation in confined compression, and, in general terms, it can be a function of the in-plane coordinates (see, e.g., [28]) and the number of cycles. An advanced constitutive model for a third-body interface was developed by Krejčí and Petrov [29] within the mathematical framework of hysteresis operators, which utilizes some material parameters like Lamé elastic constants, viscosity coefficient, and yield limit that may change during the process of motion.

Now, we arrive at the question that drives this review. How should one model the growth of the function $h(x, N)$ and the expansion of the contact region $\omega_{N}$ that occur due to wear? Taking into account that the third-body layer is formed from a part of the cumulative worn material, the latter question implies the formulation of two problems: $(i)$ the mass balance of retained and ejected worn material, (ii) the law of in-plane evolution of the third-body layer.

The question of mass balance in the wear debris layer formation was considered in a number of publications (see, e.g., [30]). In particular, a simple model derived by Fillot et al. [31], using 3D discrete element simulations, operates with the mean thickness, $H$, of the 
third body. By denoting with $\rho_{\text {tb }}$ the density of the third body, the total mass of the third body particles trapped at the contact interface can be estimated as $M=\rho_{\mathrm{tb}} A H$, where $A$ is the in-plane contact area. Then, according to [31], the global progression in time of the third body that accounts for the degradation mass flow, $Q_{\mathrm{d}}=C_{\mathrm{d}}\left(H_{\max }-H\right)$, and the ejection mass flow, $Q_{\mathrm{e}}=C_{\mathrm{e}} H$, is described by the balance equation $\mathrm{d} M / \mathrm{d} t=Q_{\mathrm{d}}-Q_{\mathrm{e}}$, from where it follows that the mean three-body thickness $H$ exponentially tends to the stable value $H_{\text {stab }}=C_{\mathrm{d}}\left(C_{\mathrm{d}}+C_{\mathrm{e}}\right)^{-1} H_{\text {max }}$, where $H_{\text {max }}$ is a so-called threshold thickness, which corresponds to the number of third particles that stops the degradation flow. It is to note here that $C_{\mathrm{d}}$ and $C_{\mathrm{e}}$ are empirical constants that drive the degradation and ejection mass flows, respectively. A shortcoming of the lumped parameter model [31], which was further developed in [32], is that it does take into account neither the advancement of the contact area nor the variability of the thickness of the third-body layer.

Strictly speaking, the density and mechanical properties of the growing third-body layer evolves during the fretting wear process. The displacement accommodation and plastification process of the third body under tangential loading was considered in [33]. A straightforward hypothesis would be that this phenomenon is similar to the shake-down effect observed under repeated loading $[34,35]$. We note here that an elasto-plastic model for the two-dimensional deformation of the third-body layer in rolling wheel/rail contact was developed in [36]. A review of earlier approaches for modelling solid third bodies in dry sliding contact was given by Iordanoff et al. [9] with an emphasis on the debris flow rheology.

A simple way to model the evolution of third body was suggested by Arnaud and Fouvry [37] (see also [38]), who introduced the concept of conversion factor, $\gamma(x, N)$, that governs the pointwise increment of the third-body layer thickness as

$$
\Delta h(x, N)=\gamma(x, N) \Delta w(x, N),
$$

where $\Delta w(x, N)$ is the total linear wear increment. Observe that both increments $\Delta w(x, N)$ and $\Delta h(x, N)$ depend on the same variables. However, while the dependence on $N$ indicates the cycle-to-cycle evolution of the wear characteristics, the dependence on the coordinate $x$ needs to be clarified. By writing Eq. (3), it is tentatively assumed that the fretting stroke, $\Delta x$, is sufficiently small to identify the "point" of wear debris generation, which produces the linear wear increment $\Delta w(x, N)$, with the corresponding point of the third-body layer. Loosely speaking, the wear debris can be regarded as "frozen" in the first bodies before the detachment moment, and therefore, the abscissa $x$ in $\Delta w(x, N)$ can be taken as a Lagrange coordinate. On the other hand, the dependence of $\Delta h(x, N)$ on the coordinate $x$ should reflect the distance to the boundary, which is traveled by the wear debris that escapes from the contact region. We note that Eq. (3) generalizes the hypothesis introduced by Goryacheva and Goryachev [39] that the thickness of the third-body layer is proportional to the depth of the worn layer. To simplify the semi-analytical fretting wear simulations with the effect of wear debris, it was suggested by Done et al. [40] to consider that the third-body layer is attached to the surface of one of the contacting bodies (presumably to that which has grater surface curvature).

Recently, Zhang et al. [41] introduced the volume fraction of ejected loose debris, $\bar{\gamma}$, as the ratio of the volume increment of the newly generated loose particles to the total volume increment of the newly worn volume $\Delta V_{N}$. In such a way, the volume increment of the trapped debris particles compacted on the third-body layer equals $\Delta V_{\mathrm{tb}}=(1-\bar{\gamma}) \Delta V_{N}$ and the corresponding increment of the third-body layer thickness is calculated as follows: 
$\Delta h(x, N)=(1-\bar{\gamma}) \Delta w(x, N)$. Obviously, the latter equation is a form of Eq. (3) with a constant conversion factor $\gamma=1-\bar{\gamma}$, which is independent of the spatial coordinates. To account for the evolution of the third-body layer, which is associated with the layer thickness, a threshold-dependence of $\bar{\gamma}$ on $h(x, N)$ was also introduced in [41].

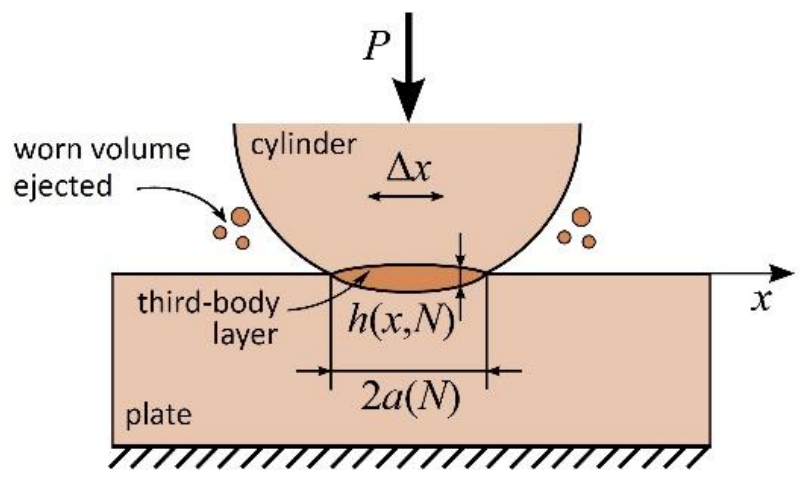

Fig. 1 Plane contact between elastic cylinder and plate with third body

Following [37], a quadratic law for the conversion factor $\gamma$ in the cylinder-on-plate contact configuration (see Fig. 1) was adopted by Argatov and Chai [42], but represented in the self-similar form, that is the factor $\gamma$ is assumed to be a function of the dimensionless ratio $x / a(N)$, where $a(N)$ is the half-width of the contact region. In the two-dimensional setting, such a quadratic law can be written as

$$
\gamma(x, N)=\gamma_{0}-\kappa_{0} \frac{x^{2}}{a^{2}(N)},
$$

where $\gamma_{0}$ and $\kappa_{0}$ are constants that may depend on $N$.

In light of (4), it can be argued [42] that after a relatively short initial period, the contact pressure density (with the exception of the boundary-layer solutions) can be described by a self-similar profile

$$
p(x, N) \cong \frac{P}{2 a(N)} f\left(\frac{x}{a(N)}\right),
$$

where the function $f(\bar{x})$ of a dimensionless variable $\bar{x}$ satisfies the normalization condition that follows from Eq. (1). Formula (5) allows to estimate the continuous variation of the third-body layer profile, thickness, and volume.

It is worth emphasizing that Eq. (3) tentatively assumes a certain law of wear, which relates the linear wear increment $\Delta w(x, N)$ to the contact pressure $p(x, N)$. Another issue that deserves further attention is related to the simplified model for the third body evolution via the conversion factor, which is described above. Apparently, a promising approach to this problem lies in applying the Kachanov-Rabotnov damage mechanics-based methodology, which was exploited by Ghosh et al. [43] in their analysis of fretting wear. 


\section{DISCUSSION AND CONCLUSION}

It goes without saying that, while a substantial progress has been made in recent years in analysis of fretting wear (without third body) both in gross-slip and partial-slip regimes as well as in two- and three-dimensional settings, the problem of third body modelling is still under-researched, in particular, in regard to modelling the in time and in-plane evolution of the third-body layer under partial-slip fretting wear conditions.

When considering the contact problem with the effect of wear, the issue of contact geometry adaptation [44] should be addressed first. The third body influences the contact pressure distribution not only via the accommodation of the contact displacements, but also via partially filling the variable gap between the surfaces of the first bodies. This means that the variable thickness of the third-body layer is an important characteristic that links the third body evolution to the geometry accommodation aspect.

Within this perspective, it looks promising to derive a transient analogue of formula (4) for the conversion factor from the previously developed theoretical considerations, which are discussed above. On the other hand, there is still a lack of modelling efforts on representing the results of numerical simulations in an analytical form that is amendable to analysis.

To conclude, one can say that the problem of analytical modelling of the third-bodylayer evolution in fretting wear contact is far from been completely understood.

Acknowledgement: This work was supported by the National Research Foundation of Korea (NRF) grant funded by the Korean government (MSIT) (No. NRF-2017M2B2A9072449).

\section{REFERENCES}

1. Vingsbo, O., Söderberg, S., 1988, On fretting maps. Wear, 126(2), pp. 131-147.

2. Chai, Y.S., Lee, C.Y., Bae, J.W., Lee, S.Y., Hwang, J.K., 2005, Finite element analysis of fretting wear problems in consideration of frictional contact, Key Engineering Materials, 297-300, pp. 1406-1411.

3. Blau, P J., 2019, A microstructure-based wear model for grid-to-rod fretting of clad nuclear fuel rods, Wear, 426, pp. 750-759.

4. Farris, T., Szolwinski, M., Harish, G., 2000, Fretting in aerospace structures and materials, In: Hoeppner, D., Chandrasekaran, V., Elliott, C. (Eds.), Fretting Fatigue: Current Technology and Practices, West Conshohocken, PA: ASTM International, 2000, pp. 523-537.

5. Godet, M., 1984, The third-body approach: a mechanical view of wear, Wear, 100(1-3), pp. 437-452.

6. Ding, J., McColl, I.R., Leen, S.B., Shipway, P.H., 2007, A finite element based approach to simulating the effects of debris on fretting wear, Wear, 263(1-6), pp. 481-491.

7. Basseville, S., Héripré, E., Cailletaud, G., 2011, Numerical simulation of the third body in fretting problems, Wear, 270(11-12), pp. 876-887.

8. Leonard, B.D., Ghosh, A., Sadeghi, F., Shinde, S., Mittelbach, M., 2014, Third body modeling in fretting using the combined finite-discrete element method, International Journal of Solids and Structures, 51(6), pp. 1375-1389.

9. Iordanoff, I., Berthier, Y., Descartes, S., Heshmat, H., 2002, A review of recent approaches for modeling solid third bodies, Journal of Tribology, 124(4), pp. 725-735.

10. Zmitrowicz, A., 2005, Wear debris: a review of properties and constitutive models, Journal of Theoretical and Applied Mechanics, 43(1), pp. 3-35.

11. Popov, V.L., 2010, Contact Mechanics and Friction, Springer, Berlin.

12. Hintikka, J., Mäntylä, A., Vaara, J., Frondelius, T., Lehtovaara, A., 2019, Stable and unstable friction in fretting contacts, Tribology International, 131, pp. 73-82.

13. Ciavarella, M., Demelio, G., 2001, A review of analytical aspects of fretting fatigue, with extension to damage parameters, and application to dovetail joints, International Journal of Solids and Structures, 38(10-13), pp. 1791-1811.

14. Chai, Y.S., Argatov, I.I., 2019, Fretting wear accumulation in partial-slip circular Hertzian contact, Mechanics Research Communications, 96, pp. 45-48.

15. Argatov, I.I., Bae, J.W., Chai, Y.S., 2020, A simple model for the wear accumulation in partial slip Hertzian contact, International Journal of Applied Mechanics, 12(7), 2050074. 
16. Hills, D., Andresen, H., 2020, Fundamentals of elastic contacts, In: Paggi M., Hills D. (Eds.), Modeling and Simulation of Tribological Problems in Technology, CISM International Centre for Mechanical Sciences (Courses and Lectures), vol. 593, pp. 1-39, Springer, Cham.

17. Galin, L.A., 1976, Contact problems of the theory of elasticity in the presence of wear, Journal of Applied Mathematics and Mechanics, 40(6), pp. 931-936.

18. Argatov, I., Tato, W., 2012, Asymptotic modeling of reciprocating sliding wear-comparison with finite-element simulations, European Journal of Mechanics-A/Solids, 34, pp. 1-11.

19. Argatov, I.I., Chai, Y.S., 2021, Fretting wear with variable coefficient of friction in gross sliding conditions, Tribology International, 153, 106555.

20. Stupkiewicz, S., Mróz, Z., 1999, A model of third body abrasive friction and wear in hot metal forming, Wear, 231(1), pp. 124-138.

21. Bozkaya, D., Müftü, S., 2008, The effects of interfacial particles on the contact of an elastic sphere with a rigid flat surface, Journal of Tribology, 130(4), 041401.

22. Li, Q., 2020, Simulation of a single third-body particle in frictional contact, Facta Universitatis-Series Mechanical Engineering, 18(4), pp. 537-544.

23. Zmitrowicz, A., 1987, A thermodynamical model of contact, friction and wear: I Governing equations, Wear, 114(2), pp. 135-168.

24. Zmitrowicz, A., 2001, Variational descriptions of wearing out solids and wear particles in contact mechanics, Journal of Theoretical and Applied Mechanics, 39(3), pp. 791-808.

25. Sauger, E., Fouvry, S., Ponsonnet, L., Kapsa, P., Martin, J.M., Vincent, L., 2000, Tribologically transformed structure in fretting, Wear, 245(1-2), pp. 39-52.

26. Aleksandrov, V.M., 1969, Asymptotic solution of the contact problem for a thin elastic layer, Journal of Applied Mathematics and Mechanics, 33(1), pp. 49-63.

27. Barber, J.R., 1990, Contact problems for the thin elastic layer, International Journal of Mechanical Sciences, 32(2), pp. 129-132.

28. Argatov, I., Mishuris, G., 2015, Contact Mechanics of Articular Cartilage Layers: Asymptotic Models, Springer, Cham.

29. Krejčí, P., Petrov, A., 2018, A mathematical model for the third-body concept, Mathematics and Mechanics of Solids, 23(3), pp. 420-432.

30. Zmitrowicz, A., 2007, Contact mechanics of wearing out solids, In: Wriggers, P., Nackenhorst, U. (Eds.), IUTAM Symposium on Computational Methods in Contact Mechanics, pp. 311-331, Springer, Dordrecht.

31. Fillot, N., Iordanoff, I., Berthier, Y., 2005, Simulation of wear through mass balance in a dry contact, Journal of Tribology, 127(1), pp. 230-237.

32. Fillot, N., Iordanoff, I., Berthier, Y., 2007, Wear modeling and the third body concept, Wear, 262(7-8), pp. 949-957.

33. Sun, Y., Berthier, Y., Fantino, B., Godet, M., 1993, A quantitative investigation of displacement accommodation in third-body contact, Wear, 165(2), pp. 123-131.

34. Fouvry, S., 2001, Shakedown analysis and fretting wear response under gross slip condition, Wear, 251(1-12), pp. $1320-1331$.

35. Williams, J.A., 2005, Wear and wear particles-some fundamentals, Tribology International, 38(10), pp. 863-870.

36. Meierhofer, A., Hardwick, C., Lewis, R., Six, K., Dietmaier, P., 2014, Third-body layer-experimental results and a model describing its influence on the traction coefficient, Wear, 314(1-2), pp. 148-154.

37. Arnaud, P., Fouvry, S., 2018, A dynamical FEA fretting wear modeling taking into account the evolution of debris layer, Wear, 412, pp. 92-108.

38. Arnaud, P., Fouvry, S., Garcin, S., 2017, A numerical simulation of fretting wear profile taking account of the evolution of third-body layer, Wear, 376, pp. 1475-1488.

39. Goryacheva, I.G., Goryachev, A.P., 2006, The wear contact problem with partial slippage, Journal of Applied Mathematics and Mechanics, 70(6), pp. 934-944.

40. Done, V., Kesavan, D., Chaise, T., Nelias, D., 2017, Semi analytical fretting wear simulation including wear debris, Tribology International, 109, pp. 1-9.

41. Zhang, L., Ma, S., Liu, D., Zhou, B., Markert, B., 2019, Fretting wear modelling incorporating cyclic ratcheting deformations and the debris evolution for Ti-6Al-4V, Tribology International, 136, pp. 317-331.

42. Argatov, I.I., Chai, Y.S., 2021, A self-similar model for fretting wear contact with the third body in gross slip, Wear, 466-467, 203562.

43. Ghosh, A., Leonard, B., Sadeghi, F., 2013, A stress based damage mechanics model to simulate fretting wear of Hertzian line contact in partial slip, Wear, 307(1-2), pp. 87-99.

44. Argatov, I. Chai, Y.S., 2020, Contact geometry adaptation in fretting wear: A constructive review, Frontiers of Mechanical Engineering, 6, 51. 